\title{
Multiband Mechanism for the Sign Reversal of Coulomb Drag Observed in Double Bilayer Graphene Heterostructures
}

\author{
M. Zarenia, ${ }^{1,2}$ A. R. Hamilton, ${ }^{3}$ F. M. Peeters, ${ }^{1}$ and D. Neilson ${ }^{1,4}$ \\ ${ }^{1}$ Department of Physics, University of Antwerp, Groenenborgerlaan 171, 2020 Antwerp, Belgium \\ ${ }^{2}$ Department of Physics and Astronomy, University of Missouri, Columbia, Missouri 65211, USA \\ ${ }^{3}$ ARC Centre of Excellence for Future Low Energy Electronics Technologies, School of Physics, \\ The University of New South Wales, Sydney, New South Wales 2052, Australia \\ ${ }^{4}$ Physics Division, Science \& Technology School, University of Camerino, 62032 Camerino, Italy
}

(Received 29 January 2018; published 17 July 2018)

\begin{abstract}
Coupled 2D sheets of electrons and holes are predicted to support novel quantum phases. Two experiments of Coulomb drag in electron-hole $(e-h)$ double bilayer graphene (DBLG) have reported an unexplained and puzzling sign reversal of the drag signal. However, we show that this effect is due to the multiband character of DBLG. Our multiband Fermi liquid theory produces excellent agreement and captures the key features of the experimental drag resistance for all temperatures. This demonstrates the importance of multiband effects in DBLG: they have a strong effect not only on superfluidity, but also on the drag.
\end{abstract}

DOI: 10.1103/PhysRevLett.121.036601

Electron-hole $(e-h)$ double-sheet van der Waals heterostructures are attracting great interest because they are predicted to support novel quantum phases. These phases include superfluidity, coupled Wigner crystals, and charge density waves [1-6]. Novel quantum phases are a major motivator for experimental studies of Coulomb drag in coupled $e-h$ sheets in GaAs double quantum wells [7,8], graphene double monolayers $[9,10]$, hybrid grapheneGaAs systems [11,12], and graphene double bilayers (DBLG) $[13,14]$. In a drag measurement, the measured quantity is the transresistivity $\rho_{D}$, the ratio of the generated voltage in the open-circuit drag sheet (2) to the current density in the drive sheet (1) [Fig. 1(a)]. In conventional momentum drag, the dragged hole is expected to travel in the same direction as the drive electron, corresponding to a positive drag resistivity $\rho_{D}>0$.

Deviations of $\rho_{D}$ from a standard Fermi-liquid $T^{2}$ temperature dependence, are commonly accepted as evidence of correlations [15] or the existence of exotic manybody phases $[16,17]$. Two recent experimental studies of $e-h$ drag in DBLG $[13,14]$ reporting large anomalous behavior in the $\rho_{D}$ have therefore attracted a lot of attention and discussion. Even more puzzling, Lee et al. [13] reported $\rho_{D}$ that reverse sign as the carrier density was decreased at low $T$. The magnitudes of the $\rho_{D}$ increase dramatically with decreasing $T$, eventually becoming comparable to the sheet resistivity. Independently, $\mathrm{Li}$ et al. [14] also reported $\rho_{D}$ that reversed sign as a function of the density for $T$ as high as $160 \mathrm{~K}$. These are completely unexpected results, with the reversal of sign in the $\rho_{D}$ a major conundrum. The $\rho_{D}$ in double GaAs quantum wells $[15,18]$ and double graphene monolayers [10], show no sign reversal and their drag mechanisms are substantially understood $[19,20]$. However, there exists no explanation for the recent DBLG results.

We show that these striking effects can be explained in detail by the multiband character of bilayer graphene (BLG) taken together with the increase in the carrier effective masses $\left(m^{*}\right)$ at low densities and $T$. Our theory of a linear screened Fermi liquid in multiband BLG produces excellent agreement with the observed structure in the $\rho_{D}$, capturing the key features of the recent experiments over the full range of $T$ and highlighting the dramatic consequences of multiband transport on the drag.

Figure 1(b) schematically depicts electron and hole BLG sheets, (1) and (2), encapsulated in insulating hexagonal boron nitride $(h-\mathrm{BN})$ to prevent tunneling between the sheets and $e-h$ recombination. Metal gates control the sheet densities $n_{1}$ and $n_{2}$. Unlike monolayer graphene, BLG has single-particle energy bands with quadratic dispersion at low energies, and its conduction band (CB) and valence band (VB) are separated by a small variable band gap induced by the perpendicular electric fields from the gates. In contrast, monolayer graphene has linearly dispersing bands with no band gap, while GaAs has quadratic bands with a fixed large band gap $\gg k_{B} T$. We find in DBLG that there is significant multiband transport in both the CB and VB of each sheet which must be included in calculations. An understanding of the nature of the multiband mechanisms lying behind these surprising experimental results paves the way for future drag experiments. Multiband effects have already been shown to be important for $e-h$ superfluidity in coupled 2D materials [21]. 


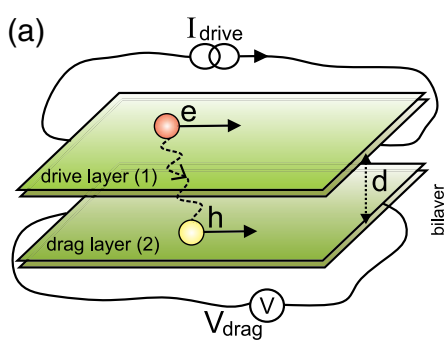

(c) Experiment: Ref. [14]

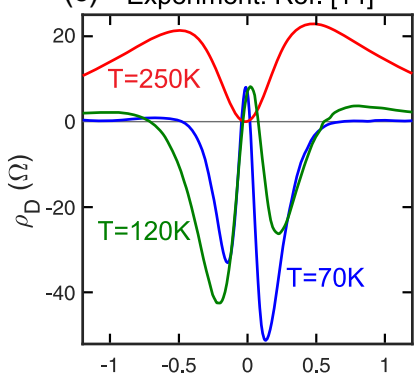

(e) Experiment: Ref. [13]
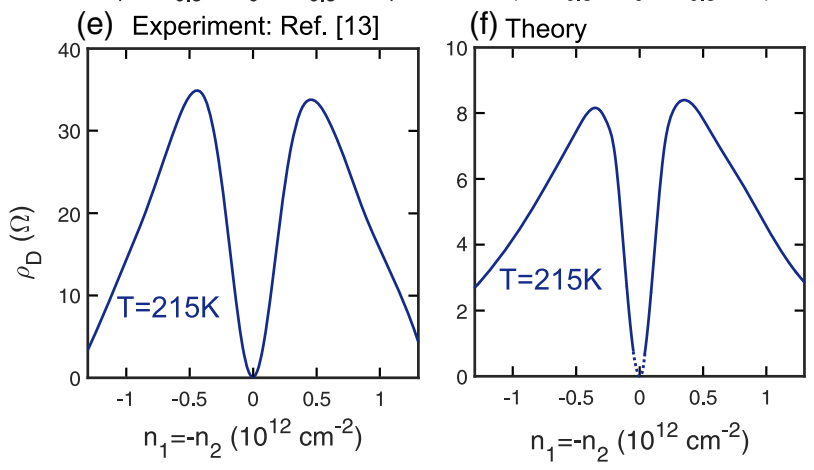

FIG. 1. (a) Schematic representation of the $e-h$ drag measurement. (b) Schematic of the device. (c) Experimental drag measurements from Ref. [14]. (d) Our calculated drag for sample in Ref. [14]. (e) Experimental data from sample $A$ of Ref. [13]. (f) Our calculated drag for sample $A$ in Ref. [13].

Figures 1(c)-1(f) compare our $\rho_{D}$ at fixed $T$ as a function of matched densities $n_{1}=-n_{2}$ with the data of Refs. [13,14]. Figure 1(c) shows data from Ref. [14] of the drag. For all $T$, the system displays conventional positive drag at high densities, but unexpected sharp negative peaks appear as the density is reduced at lower $T$.

Figure 1(d) shows our calculated $\rho_{D}$ for the experimental parameters in Ref. [14]: $h$-BN barrier thickness $d=5.2 \mathrm{~nm}$; the transverse electric fields at the dual neutrality point (DNP) due to unintentional doping are not precisely specified and we take reasonable values, $\left(E_{1}^{0}, E_{2}^{0}\right)=$ $(0.05,0) \mathrm{meV} \mathrm{nm}^{-1}$. Our results in Fig. 1(d) capture the key structure of the unusual positive and negative peaks in the experimental $\rho_{D}$ at fixed $T$, as well as the change of sign of the $\rho_{D}$ when $T$ is increased. The asymmetries in the negative peaks in Figs. 1(c) and 1(d) are reversed. We will see later [Fig. 3(b)] that, unlike the positions of the peaks, their asymmetries are very sensitive to $E_{1}^{0}$ and $E_{2}^{0}$, and small changes can reverse the asymmetry. Lacking precise experimental values for $E_{1}^{0}$ and $E_{2}^{0}$, we chose not to attempt to reproduce the asymmetries through small adjustments of $E_{1}^{0}$ and $E_{2}^{0}$.

Consistent with experiment, we obtain positive $e-h$ drag for $T=215$ and $250 \mathrm{~K}$, but strong negative drag at small densities for $T=70$ and $120 \mathrm{~K}$. A discrepancy is that our theory does not reproduce the small positive peak in $\rho_{D}$ observed at the DNP for $T=70$ and $120 \mathrm{~K}$. This peak is known to be associated with energy drag from $e$ - $h$ puddle density fluctuations not considered by our theory. Our $\rho_{D}$ must vanish at the DNP, and very close to the DNP we extrapolate $\rho_{D}$ to zero (dotted lines). Our theory also reproduces the key features of drag measured in different samples in another laboratory. Figure 1(e) shows data from sample $A$ of Ref. [13], and Fig. 1(f) the calculated drag using the experimental parameters $d=3 \mathrm{~nm}$ and $\left(E_{1}^{0}, E_{2}^{0}\right)=(0.1,-0.04) \mathrm{meV} \mathrm{nm}^{-1}$.

We use a well-established procedure for determining the drag transresistivity $\rho_{D}$ in $e-h$ DBLG at fixed $T$ as a function of equal densities, $n_{1}=-n_{2}$. We use the random phase approximation (RPA) linear-response screening theory for Fermi liquids in the clean limit. The consistent underestimate of the magnitude of $\rho_{D}$ in our model may be due to many-body correlations, which are expected to increase the magnitude of $\rho_{D}$ but not to strongly modify its overall shape [15]. New in our approach is the inclusion of the induced variable band gaps between the conduction and valence bands of the bilayers, the link between the variable band gaps and the carrier densities, and the increase in $m^{*}$ near the DNP at low $T$.

$\rho_{D}$ is related to the drag conductivity $\sigma_{D}$ by $[19,22]$

$$
\rho_{D}=-\sigma_{D} /\left[\sigma_{1} \sigma_{2}-\sigma_{D}^{2}\right] \simeq-\sigma_{D} / \sigma_{1} \sigma_{2} .
$$

$\sigma_{i}=n_{i} e^{2} \tau_{i} / m^{*}$ is the longitudinal conductivity for sheet $(i)$ with momentum independent scattering time, $\tau_{1} \approx \tau_{2} \equiv \tau$. Charge impurity scattering and short-range impurity scattering both lead to transport scattering times independent of momentum in BLG [20]. When there are transverse electric fields at the DNP from unintentional doping, $m^{*}$ is expected to be enhanced at low densities [23], as recently observed experimentally [13,24].

$\sigma_{D}$ is a convolution of the density fluctuations within the sheets represented by $\operatorname{Im} \chi_{i}(\boldsymbol{q}, \omega), i=1,2$, the imaginary part of the nonlinear susceptibility of each sheet,

$$
\sigma_{D}=\frac{\hbar}{4 \pi k_{B} T} \int d^{2} \boldsymbol{q} d \omega \frac{\left|V_{12}(\boldsymbol{q}, \omega)\right|^{2} \operatorname{Im} \chi_{1}(\boldsymbol{q}, \omega) \operatorname{Im} \chi_{2}(\boldsymbol{q}, \omega)}{\sinh ^{2} \hbar \omega / 2 k_{B} T},
$$

$$
\begin{aligned}
& \operatorname{Im} \chi_{i}(\boldsymbol{q}, \omega) \\
& \quad=e \tau \sum_{\gamma \gamma^{\prime}} \operatorname{Im}\left(\int d^{2} \boldsymbol{k} \frac{F_{\boldsymbol{k}, \boldsymbol{q}}^{\gamma \gamma^{\prime}}\left(f_{\epsilon_{\boldsymbol{k}, \gamma}}^{i}-f_{\epsilon_{\boldsymbol{k}+\boldsymbol{q} \gamma^{\prime}}}^{i}\right)\left(v_{\boldsymbol{k}, \gamma}^{x, i}-v_{\boldsymbol{k}+\boldsymbol{q}, \gamma^{\prime}}^{x, i}\right)}{\varepsilon_{\boldsymbol{k}, \gamma}^{i}-\varepsilon_{\boldsymbol{k}+\boldsymbol{q}, \gamma^{\prime}}^{i}+\hbar \omega+i 0^{+}}\right) .
\end{aligned}
$$


$\gamma=C, V$ labels the CB and VB. The form factors $F_{k, q}^{\gamma \gamma^{\prime}}$ come from the overlap of the wave functions in the gapped BLG [25]. In band $\gamma$ of sheet $(i), f_{\varepsilon_{k, \gamma}}^{i}=1 /\left[e^{\left(\epsilon_{k, \gamma}-\mu_{i}\right) / k_{B} T}+1\right]$ is the Fermi-Dirac function with chemical potential $\mu_{i}, v_{\boldsymbol{k}, \gamma}^{x, i}$ is the $x$ component of the velocity, and $\varepsilon_{\boldsymbol{k}, \gamma}^{i}$ is the singleparticle energy, obtained using the four-band Hamiltonian for biased BLG subbands [26] with variable band gap $E_{g}^{i}$. For the small gaps we work with, $E_{g}^{i}<20 \mathrm{meV}$, and for the low densities, $n=p<10^{12} \mathrm{~cm}^{-2}$, our results are not changed significantly if we use the quadratic energy dispersion obtained with the corresponding two-band Hamiltonian.

In the $|\mu| \rightarrow 0$ limit, the analytic solutions for $\rho_{D}$ in a clean system become numerically tedious to solve, and since $\rho_{D} \rightarrow 0$ for $|\mu| / k_{B} T \ll 1$, we extrapolate it to zero in this limit. This extrapolation may not even be relevant to experiments, since it will be masked by the peak in $\rho_{D}$ due to disorder-induced $e-h$ puddle density fluctuations [27] that are absent in the clean system.

The band gap $E_{g}^{i}$ in sheet $(i)$ depends on the transverse electric fields $E_{i}[28,29]$ from the metallic gates. Thus, $E_{g}^{i}$ changes slightly with the carrier density $n_{i}$. Through the doping of each bilayer, $E_{i}$ can be described as (see the Supplemental Material in Ref. [13]),

$$
E_{i}=\delta_{i 2}\left(e n_{2} / 2 \epsilon_{0}\right)+e n_{1} / \epsilon_{0}+E_{i}^{0} .
$$

According to the experimental data from Refs. [24,29], the induced band gap $E_{g}^{i} \approx \alpha E_{i}$, with $\alpha \sim 0.1 e \mathrm{C} \mathrm{nm}$ for weak $E_{i}$, and $E_{g}^{i}$ and $E_{i}$ in $\mathrm{meV}$ and $\mathrm{mV} \mathrm{nm}^{-1}$. Typically in the samples in Refs. [13,14], $E_{g}^{i} \lesssim 20 \mathrm{meV}$.

At low $T$ when the system is degenerate, we take a typical value for the gap at the DNP of $E_{g}^{i}=20 \mathrm{meV}$ to calculate the enhancement of $m^{*}$ at small densities, using the density-dependent expression for $m^{*}$ in Ref. [30]. At room temperature, the number of thermally excited carriers will be large enough to suppress this enhancement. Hence, we interpolate $m^{*}$ from the density-dependent values for low $T$ to the unrenormalized, constant value $m^{*}=0.04 m_{e}$ [31] at room temperature.

The dynamically screened Coulomb interaction between sheets $(i)$ and $(j)$ within the RPA is $V_{i j}(\boldsymbol{q}, \omega)=$ $(-1)^{i-j} v(q) \exp \left[-q d\left(1-\delta_{i j}\right)\right] / \operatorname{det}|\epsilon(\boldsymbol{q}, \omega)|$, where $v(q)=$ $2 \pi e^{2} / \kappa q$ is the bare Coulomb interaction and $\epsilon_{i j}(\boldsymbol{q}, \omega)=\delta_{i j}+V_{i j}(\boldsymbol{q}, \omega) \Pi_{i}(\boldsymbol{q}, \omega)$, with $\Pi_{i}(\boldsymbol{q}, \omega)$ the RPA polarization function for sheet $(i)$ [25]. At low electric fields, $E_{i} \lesssim 10 \mathrm{meVnm}^{-1}$, the $\kappa$ for an isolated BLG encapsulated in $h$-BN layers increases from 2 for a few layers to 4 for many layers [32]. For our DBLG encapsulated in $h$-BN, we expect $2 \lesssim \kappa \lesssim 4$. Across this range of $\kappa$, we find the shape of the drag peaks in $\rho_{D}$ does not significantly vary, but their height increases with increasing $\kappa$ [the strength of screening in $V_{i j}(\boldsymbol{q}, \omega)$ depends on $\kappa$ ]. For our results we have set $\kappa=4$.
We numerically solve Eqs. (1)-(3) for $\rho_{D}$. We fix the chemical potential $\mu_{i}$ for sheet (i) using

$$
n_{i}=4 \int d^{2} \boldsymbol{k}\left[f_{\varepsilon_{k, \gamma=1}}^{i}+\left(f_{\varepsilon_{k, \gamma=-1}}^{i}-1\right)\right] .
$$

To understand the origins of the drag response structure, in Fig. 2 we examine the four terms in the summation in Eq. (3) over the CB and VB, $\gamma=C, V$ and $\gamma^{\prime}=C, V$. For Fig. 2 we set $T=70 \mathrm{~K}$, comparable to the band gaps $E_{g}^{1}=E_{g}^{2}$ that correspond to $E_{1}^{0}=-E_{2}^{0}=0.05 \mathrm{meV} \mathrm{nm}^{-1}$. Figure 2(a) shows schematically the evolution of the $\mathrm{CB}$ and VB structure with density in the $e$-doped drive bilayer (1) and $h$-doped drag bilayer (2). Unlike conventional semiconductors, the band gaps are small and depend on density. At higher densities, the chemical potentials $\mu_{i}$ (red lines) are in the CB and VB, leading to asymmetric band populations, schematically represented in the left panels of Fig. 2(b). The large contributions to the summations in Eq. (3) are the intraband terms from the CB in bilayer (1), $\operatorname{Im} \chi_{1}^{C C}$ [blue curve in right panels of Fig. 2(b)], and the VB in bilayer (2), $\operatorname{Im} \chi_{2}^{V V}$ (green curve). Since $\operatorname{Im} \chi_{1}^{C C}$ and $\operatorname{Im} \chi_{2}^{V V}$ have opposite signs, the total $\operatorname{Im} \chi_{1}(\boldsymbol{q}, \omega)$ and $\operatorname{Im} \chi_{2}(\boldsymbol{q}, \omega)$ (black curves) also have opposite signs. The $e-h \operatorname{drag} \rho_{D} \propto-\left(\operatorname{Im} \chi_{1} \operatorname{Im} \chi_{2}\right)$ is then everywhere positive, as in a conventional semiconductor.

Figure 2(c) is at a smaller density where the $\mu_{i}$ lie near the midgaps. Thermal fluctuations ensure there are significant carrier populations in the CB and VB [left panels Fig. 2(c)], which makes each term in Eq. (3) similar for the two sheets, $\operatorname{Im} \chi_{1}^{\gamma \gamma^{\prime}} \approx \operatorname{Im} \chi_{2}^{\gamma \gamma^{\prime}}$ [compare the curves in the right panels of Fig. 2(c)]. Since $\operatorname{Im} \chi_{1}(\boldsymbol{q}, \omega)$ and $\operatorname{Im} \chi_{2}(\boldsymbol{q}, \omega)$ (black curves) are similar and have the same sign, $\rho_{D} \propto$ $-\left(\operatorname{Im} \chi_{1} \operatorname{Im} \chi_{2}\right)$ is negative. This is the key mechanism behind the sign change in $\rho_{D}$. A change of sign occurs with increasing density when $\mu$ moves from the midgap to one of the bands, and it thus represents a crossover from multiband to single-band physics.

At higher $T$ (not shown), the $\mu_{i}$ evolve with increasing density similarly to Fig. 2(a). However, this evolution occurs much faster because of the smaller $m^{*}$ at higher $T$. For $T \gtrsim 200 \mathrm{~K}$, the negative peaks in $\rho_{D}$ are confined to such an extremely narrow band of densities around the DNP that they would be practically unobservable due to disorder, and the $\rho_{D}$ is essentially everywhere positive. We note that the renormalization of $m^{*}$ at low $T$ is essential for the negative peaks; if $m^{*}$ is not renormalized, the negative drag in Fig. 1(d) for $T=70$ and $120 \mathrm{~K}$ would similarly be unobservable, being narrowly confined around the DNP.

Figure 3(a) shows the evolution of $\rho_{D}$ for different $T$ for barrier thickness $d=5.2 \mathrm{~nm}$ and $E_{1}^{0}=-E_{2}^{0}=0.05 \mathrm{Vnm}^{-1}$. Note for $T \lesssim 30 \mathrm{~K}$ we predict $\rho_{D}$ will be everywhere positive, since thermal excitations are small. When $T$ increases, the thermal excitations make the bilayers nearly intrinsic at lower densities [see Fig. 2(c)], while at higher densities, the 

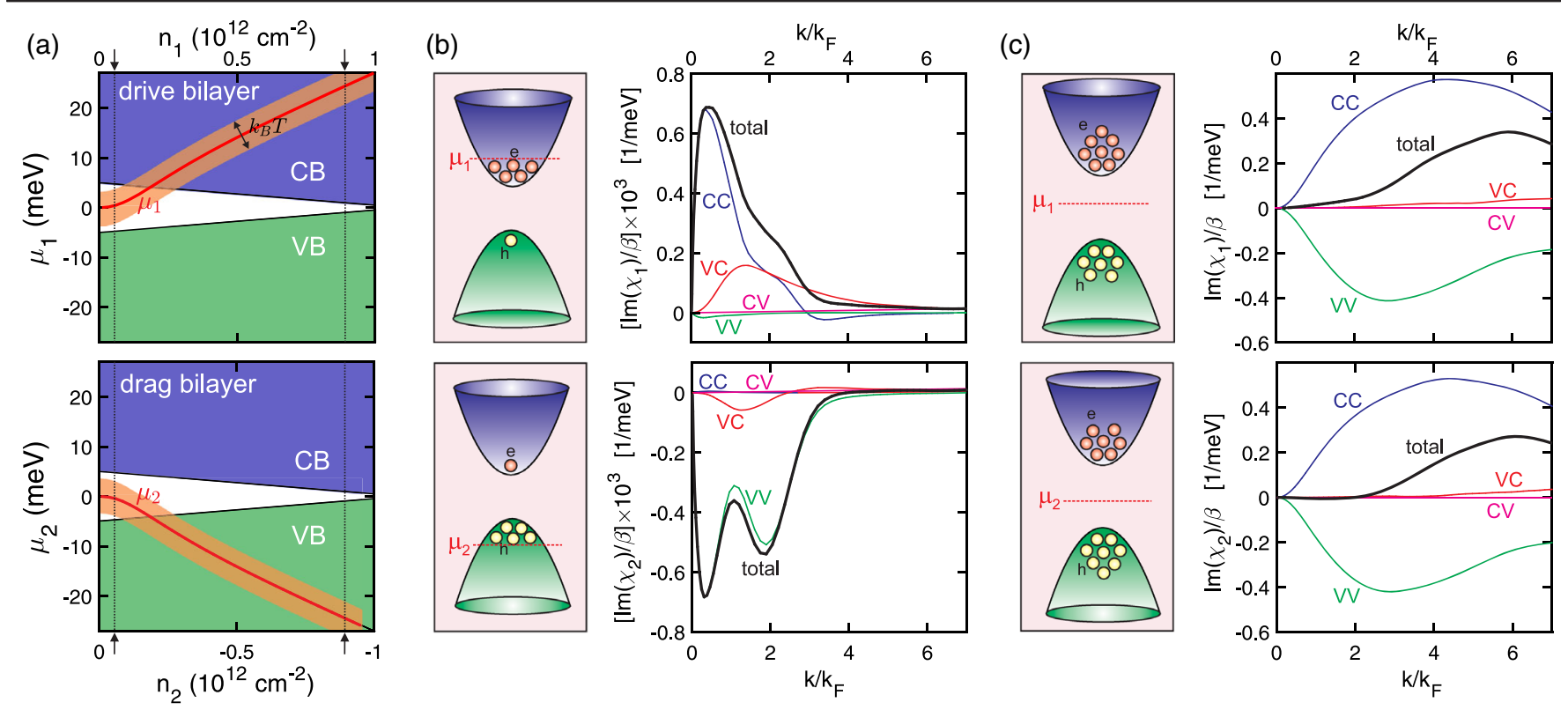

FIG. 2. Physics behind the change of sign in $\rho_{D}$. We set $T=70 \mathrm{~K}$. (a) Evolution of the bands with density in the $e$ and $h$ bilayers (1) and (2). The orange areas represent thermal excitations. (b) Left panels: carrier populations for a higher density $n_{1}=-n_{2}=0.8 \times 10^{12} \mathrm{~cm}^{-2}$. Right panels: contributions in Eq. (3), $\operatorname{Im} \chi_{1}^{\gamma \gamma^{\prime}}(\boldsymbol{q}, \omega)$ and $\operatorname{Im} \chi_{2}^{\gamma \gamma^{\prime}}(\boldsymbol{q}, \omega)$ (colored curves), for $\hbar \omega=1 \mathrm{meV}$. $\beta=e \tau \hbar k_{F}^{3} /\left(2 m^{*}\right)$. Dominant contributions $\operatorname{Im} \chi_{1}^{C C}$ and $\operatorname{Im} \chi_{2}^{V V}$ have opposite signs so $\rho_{D} \propto-\left(\operatorname{Im} \chi_{1} \operatorname{Im} \chi_{2}\right)>0$. (c) Left panels: the comparable CB and VB populations for lower density $n_{1}=-n_{2}=0.05 \times 10^{12} \mathrm{~cm}^{-2}$. Right panels: contributions to Im $\chi_{1}^{\gamma \gamma^{\prime}}(\boldsymbol{q}, \omega)$ and $\operatorname{Im} \chi_{2}^{\gamma \gamma^{\prime}}(\boldsymbol{q}, \omega)$. The terms for each $\left(\gamma \gamma^{\prime}\right)$ are similar for both sheets, so $\rho_{D} \propto-\left(\operatorname{Im} \chi_{1} \operatorname{Im} \chi_{2}\right)<0$.

$\mu_{i}$ have moved to the $\mathrm{CB}$ and $\mathrm{VB}$, resulting in asymmetric band populations and a positive $\rho_{D}$ [see Fig. 2(b)]. For $T \gtrsim 250 \mathrm{~K}$, the reduction in $m^{*}$ away from the DNP drives the $\mu_{i}$ rapidly to the $\mathrm{CB}$ or $\mathrm{VB}$, again resulting in asymmetric band populations and a positive $\rho_{D}$. The inset shows the $T$ dependence of $\rho_{D}$ at fixed densities. Consistent with the experimental data in the inset of Fig. 2(c) in Ref. [14], at low densities $\rho_{D}$ changes sign: from positive at small $T$ to negative at intermediate $T$, and back to positive as $T$ approaches room temperature. At higher densities, the $\rho_{D}$ is positive for all $T$ because the $\mu_{i}$ are in the CB and VB, making the interband contributions negligible.

Figure 3(b) highlights the sensitivity of the drag to the transverse electric fields $E_{i} . \rho_{D}$ is shown at $T=70 \mathrm{~K}$ for different combinations of $E_{i}^{0}$. The barrier thickness $d=5.2 \mathrm{~nm}$. The maximum and minimum $\rho_{D}$ both increase with $E_{i}^{0}$. When the $E_{i}$ (and hence the band gaps) are sufficiently large, $k_{B} T<\mu_{i}+E_{i}^{g}$, and $\rho_{D}$ is positive for all densities, since thermal excitations are negligible. When the $E_{1}^{0} \neq E_{2}^{0}$, the $\rho_{D}$ is asymmetric upon interchanging the $e$ - and $h$-doped sheets. The inset shows the amplitudes of the positive and negative peaks for fixed $E_{1}^{0}$ as a function of $E_{2}^{0}$. The drag maximum $\rho_{D}^{\max }$ and minimum $\rho_{D}^{\min }$ both increase with $E_{g}^{i=2}$. At very large $E_{2}^{0}$, the $\rho_{D}^{\max }$ saturates. A one-band analysis is then sufficient. The drag becomes entirely positive when $E_{2}^{0} \gtrsim 0.1 \mathrm{meV} \mathrm{nm}^{-1}$. Thus, while the change in sign of $\rho_{D}$ is relatively robust, when the asymmetry of the two band gaps becomes too pronounced, the sign change is eventually lost. By tuning the initial band gaps in the graphene bilayers $E_{i}^{0}$ with respect to the thermal excitation energy, the sign of the drag at a fixed density and $T$ can be reversed.

No sign reversal of drag has been observed for $e-h$ graphene monolayers or GaAs double quantum wells. For graphene monolayers away from the DNP, the $\mu_{i}$ move (a)

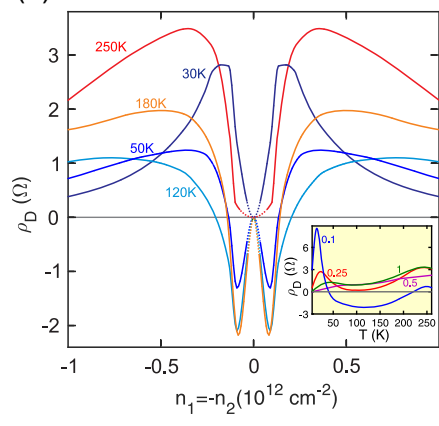

(b)

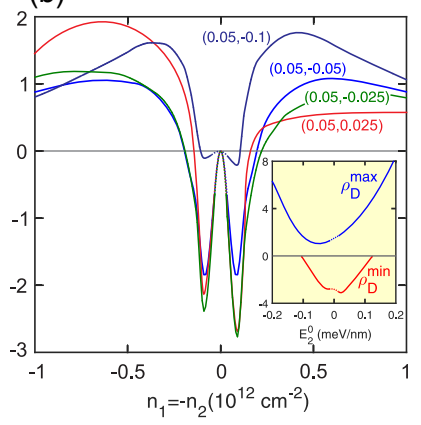

FIG. 3. (a) Evolution with $T$ of the sign reversal and peaks of $\rho_{D}$. For $50 \leq T \leq 180 \mathrm{~K}$ the bilayers are almost intrinsic at low densities [see Fig. 2(c)] resulting in a negative drag peak, while at higher densities the band populations are asymmetric [Fig. 2(b)], making $\rho_{D}$ everywhere positive. The inset shows the $T$ dependence of $\rho_{D}$. Curve labels are densities in $10^{12} \mathrm{~cm}^{-2}$. (b) Sensitivity of $\rho_{D}$ to the transverse electric fields $E_{i}$. Curve labels are the fields at the DNP, $\left(E_{1}^{0}, E_{2}^{0}\right)$ in $\mathrm{meV} \mathrm{nm}^{-1} . T=70 \mathrm{~K}$. The inset shows the magnitudes, in $\Omega$, of the negative and positive peaks $\left(\rho_{D}^{\min }, \rho_{D}^{\max }\right)$ as functions of $E_{2}^{0}$ for fixed $E_{1}^{0}=0.05 \mathrm{meV} \mathrm{nm}^{-1}$. 
rapidly to the $\mathrm{CB}$ and $\mathrm{VB}$, and so negative drag peaks would only occur so close to the DNP that they would be undetectable. For GaAs the large band gap of $\sim 1.5 \mathrm{eV}$ means $k_{B} T \ll E_{g}^{i}$ always, so the $\mu_{i}$ remain near the CB and VB. Then, the $\operatorname{Im} \chi_{1}(\boldsymbol{q}, \omega)$ and $\operatorname{Im} \chi_{2}(\boldsymbol{q}, \omega)$ have opposite signs, leading to positive $\rho_{D}$.

The main limitation of our approach is that our calculations are made within RPA in the clean limit. Our agreement with the experimental peak structures of the $\rho_{D}$ indicates that the effects of disorder and correlations can have no major impact on the position of the $\rho_{D}$ peaks at fixed $T$. Possible effects of superfluidity $[5,33]$ on the drag would occur at much lower $T$ than we consider.

This work reveals a new mechanism of drag in coupled multiband 2D sheets with small band gaps, and our multiband theory predicts negative drag not only in DBLG but also in related systems. Our theory shows that for small band gap systems, multiband effects have a dramatic effect on the drag even for a Fermi liquid. The reversals of sign in $\rho_{D}$ observed in Refs. $[13,14]$ as high as $T \sim 200 \mathrm{~K}[14]$ suggest that correlation effects, $e-h$ puddles, or superfluidity are not the primary mechanisms for the observed anomalous drag. The structure in the drag from multiband effects needs to be fully mapped out. Correlations, $e-h$ puddles, and superfluidity should be studied in the presence of multiband effects in BLG, in trilayer graphene, and in other new 2D materials with small or zero band gaps. Our theory also predicts that hybrid systems containing one small and one large band gap material could also exhibit changes of sign in $\rho_{D}$, as recently observed in a BLG-GaAs hybrid system [12].

We are grateful to Cory Dean, Emanuel Tutuc, and their research groups for discussing details of their experiments with us. This work was partially supported by the Flemish Science Foundation (FWO-Vl), the Methusalem program of the Flemish government, and the Australian Government through the Australian Research Council Centre of Excellence in Future Low-Energy Electronics Technologies (Project No. CE170100039). D. N. acknowledges support from the University of Camerino FAR project CESEMN.

[1] Y. E. Lozovik and V. Yudson, Zh. Eksp. Teor. Fiz. 71, 738 (1976) [Sov. Phys. JETP 44, 389 (1976)], http://www.jetp .ac.ru/cgi-bin/e/index/e/44/2/p389?a=list.

[2] J. P. Eisenstein and A. H. MacDonald, Nature (London) 432, 691 (2004).

[3] L. Świerkowski, D. Neilson, and J. Szymański, Phys. Rev. Lett. 67, 240 (1991).

[4] S. De Palo, F. Rapisarda, and G. Senatore, Phys. Rev. Lett. 88, 206401 (2002).

[5] A. Perali, D. Neilson, and A. R. Hamilton, Phys. Rev. Lett. 110, 146803 (2013).

[6] M. Zarenia, D. Neilson, and F. M. Peeters, Sci. Rep. 7, 11510 (2017).
[7] A. F. Croxall, K. Das Gupta, C. A. Nicoll, M. Thangaraj, H. E. Beere, I. Farrer, D. A. Ritchie, and M. Pepper, Phys. Rev. Lett. 101, 246801 (2008).

[8] J. A. Seamons, C. P. Morath, J. L. Reno, and M. P. Lilly, Phys. Rev. Lett. 102, 026804 (2009).

[9] S. Kim, I. Jo, J. Nah, Z. Yao, S. Banerjee, and E. Tutuc, Phys. Rev. B 83, 161401 (2011).

[10] R. V. Gorbachev, A. K. Geim, M. I. Katsnelson, K. S. Novoselov, T. Tudorovskiy, I. V. Grigorieva, A. H. MacDonald, S. V. Morozov, K. Watanabe, T. Taniguchi, and L. A. Ponomarenko, Nat. Phys. 8, 896 (2012).

[11] A. Gamucci, D. Spirito, M. Carrega, B. Karmakar, A. Lombardo, M. Bruna, L. N. Pfeiffer, K. W. West, A. C. Ferrari, M. Polini, and V. Pellegrini, Nat. Commun. 5, 5824 (2014).

[12] P. Simonet, S. Hennel, H. Overweg, R. Steinacher, M. Eich, R. Pisoni, Y. Lee, P. Märki, T. Ihn, K. Ensslin, M. Beck, and J. Faist, New J. Phys. 19, 103042 (2017).

[13] K. Lee, J. Xue, D. C. Dillen, K. Watanabe, T. Taniguchi, and E. Tutuc, Phys. Rev. Lett. 117, 046803 (2016).

[14] J. Li, T. Taniguchi, K. Watanabe, J. Hone, A. Levchenko, and C. Dean, Phys. Rev. Lett. 117, 046802 (2016).

[15] L. Śfiwierkowski, J. Szymański, and Z. W. Gortel, Phys. Rev. Lett. 74, 3245 (1995).

[16] G. Vignale and A. H. MacDonald, Phys. Rev. Lett. 76, 2786 (1996).

[17] D. K. Efimkin and V. Galitski, Phys. Rev. Lett. 116, 046801 (2016).

[18] T. J. Gramila, J. P. Eisenstein, A. H. MacDonald, L. N. Pfeiffer, and K. W. West, Phys. Rev. Lett. 66, 1216 (1991).

[19] B. N. Narozhny and A. Levchenko, Rev. Mod. Phys. 88, 025003 (2016).

[20] E. Hwang, R. Sensarma, and S. D. Sarma, Phys. Rev. B 84, 245441 (2011).

[21] S. Conti, A. Perali, F. M. Peeters, and D. Neilson, Phys. Rev. Lett. 119, 257002 (2017).

[22] A. G. Rojo, J. Phys. Condens. Matter 11, R31 (1999).

[23] E. McCann and V. I. Fal'ko, Phys. Rev. Lett. 96, 086805 (2006).

[24] K. Lee, B. Fallahazad, J. Xue, D. C. Dillen, K. Kim, T. Taniguchi, K. Watanabe, and E. Tutuc, Science 345, 58 (2014).

[25] X.-F. Wang and T. Chakraborty, Phys. Rev. B 81, 081402 (2010).

[26] E. McCann and M. Koshino, Rep. Prog. Phys. 76, 056503 (2013).

[27] J. C. W. Song and L. S. Levitov, Phys. Rev. Lett. 109, 236602 (2012)

[28] E. V. Castro, K. S. Novoselov, S. V. Morozov, N. M. R. Peres, J. M. B. L. dos Santos, J. Nilsson, F. Guinea, A. K. Geim, and A. H. C. Neto, Phys. Rev. Lett. 99, 216802 (2007).

[29] Y. Zhang, T. T. Tang, C. Girit, Z. Hao, M. C. Martin, A. Zettl, M. F. Crommie, Y.R. Shen, and F. Wang, Nature (London) 459, 820 (2009).

[30] E. McCann, Phys. Rev. B 74, 161403 (2006).

[31] K. Zou, X. Hong, and J. Zhu, Phys. Rev. B 84, 085408 (2011).

[32] P. Kumar, Y. S. Chauhan, A. Agarwal, and S. Bhowmick, J. Phys. Chem. C 120, 17620 (2016).

[33] M. Zarenia, A. Perali, D. Neilson, and F. Peeters, Sci. Rep. 4, 7319 (2014). 